\title{
Evaluación del nivel de aceptación de la metodología de docencia inversa entre los alumnos de la UPV
}

\author{
Lina Montuori ${ }^{a}$, Manuel Alcázar-Ortega ${ }^{b}$, Carlos Vargas-Salgado ${ }^{b}$ y Paula Bastida-Molina ${ }^{c}$ \\ ${ }^{a}$ Departamento de Termodinámica Aplicada, Universitat Politècnica de València, Camino de Vera, $\mathrm{s} / \mathrm{n}$, edifício 5J, $2^{\mathrm{a}}$ \\ planta.46022 Valencia (España), lmontuori@upvnet.upv.es \\ ${ }^{\mathrm{b}}$ Departamento de Ingeniería Eléctrica, Universitat Politècnica de València, Camino de Vera, s/n, edificio 5E, $2^{\mathrm{a}}$ \\ planta.46022 Valencia (España), malcazar@iie.upv.es, carvarsa@upvnet.upv.es
c Instituto de Ingeniería Energética, Universitat Politècnica de València, Camino de Vera, s/n, edificio 8E, acceso F, $5^{a}$ planta. 46022 Valencia (España), paubasmo@etsid.upv.es

\begin{abstract}
Flip Teaching, also called Flipped Classroom, is a new methodological form of development of teaching with an approach based on inverting the traditional scheme of the learning process and giving greater prominence to students. Flip Teaching has the objective of increasing student engagement and improving their conceptual understanding, combining direct instruction with constructivist methods using ICT tools, thus guaranteeing a better transfer of knowledge and experiences from teachers to students. In recent years, interest in the Flip Teaching methodology at the university level has been growing. More and more institutions have begun to implement a Flip Teaching project and among them, the Polytechnic University of Valencia stands out. In this context, the present article is aimed to investigate the level of acceptance of the Flip Teaching methodology among UPV students, identifying the barriers that hinder its implementation, as well as the factors that could favor its correct development.
\end{abstract}

Keywords: Flip-teaching, learning, methodology, competences, training

\section{Resumen}

La docencia inversa, también llamada clase invertida, es una nueva forma metodológica de desarrollo de la actividad docente con un enfoque basado en invertir el esquema tradicional del proceso de aprendizaje y de dar mayor protagonismo al alumnado. La docencia inversa tiene el objetivo de incrementar el compromiso del estudiante y de mejorar su comprensión conceptual, combinando la instrucción directa con métodos constructivistas a través del empleo de herramientas TIC, garantizando asi un mejor trasvase de conocimientos y experiencias desde el profesorado hacia al alumnado. En los últimos años, el interés hacia la metodología de clase invertida a nivel de los estudios universitarios ha sido creciente. Cada vez más instituciones han empezado a implantar proyecto de docencia inversa y entre ellas destaca también la Universidad Politécnica de Valencia. En este contexto, se desarrolla el presente artículo, que pretende investigar el nivel de aceptación de la metodología de docencia inversa entre los alumnos de la $U P V$, identificando las barreras que obstaculizan su implementación, asi como los factores que podrían favorecen su correcta difusión.

Palabras clave: Docencia inversa, aprendizaje, metodología, competencias, formación. 


\section{Introducción}

La Docencia Inversa (DI), también llamada Clase Invertida, es una nueva forma metodológica de Desarrollo de la actividad docente, con un enfoque novedoso de trasmitir conocimientos que se basa en invertir el esquema tradicional del proceso de aprendizaje y de protagonizar el alumnado (Montanes, et al., 2018).

Se trata de un enfoque integral que incrementa el compromiso del estudiante y pretende mejorar su comprensión conceptual, combinando la instrucción directa con métodos constructivistas a través del empleo de herramientas TIC (The Flipped Classroom, 2015). Esta metodología de docencia apoya todas las fases de un ciclo de aprendizaje de acuerdo con la Taxonomía de Bloom y fomenta el desarrollo de competencias transversales, además de las específicas (Roach, 2014), (Bonet, et al., 2015).

Una de las primeras aplicaciones de métodos de clase invertida aparece en 1871. En la Escuela de Leyes de Harvard, el decano Christopher C. Langdell implantó una metodología de aprendizaje que consistía en el estudio en casa de casos de derecho y de ley por parte de los alumnos para después discutirlos en aula, lo que conseguía desarrollar el pensamiento crítico de abogados y juristas preparándose para discutirlos en aulas desarrollando así el pensamiento crítico típico de abogados y juristas (Christensen, et al., 1992).

Actualmente, entre las aplicaciones más exitosas de DI, cabe mencionar el caso de la escuela Clintondale High School en Michigan (USA). En 2010, esta escuela estuvo entre las primeras en aplicar la metodología de clase invertida. El director de la escuela solía utilizar esta metodología para enseñar el béisbol a su hijo de 11 años. La aplicación en la escuela le permitió mejorar su posicionamiento en el ranking de los centros, disminuyendo el fracaso escolar en todas las áreas (Aulaplaneta, 2015).

A nivel de estudios universitarios, múltiples trabajos de investigación han documentado los potenciales beneficios que la implantación de la metodología de DI puede conllevar, calificándola como metodología emergente para sustituir las clases tradicionales. En los últimos años, un número creciente de instituciones ha empezado a implantar proyectos de DI y entre ellas se encuentra también la Universidad Politécnica de Valencia (UPV). Junto con el proyecto de competencias transversales, el proyecto de DI apunta a formar alumnos para que adquieran no solo competencias específicas, sino también competencias transversales que puedan introducirlos con éxito en el mundo del trabajo.

En el marco del proyecto de DI de la UPV, en 2014-2015 (Argente, et al., 2015) llevaron a cabo un exitoso proyecto piloto de implantación de docencia inversa con alumnos del segundo curso del Grado en Ingeniería Informática (Llácer-Iglesias \& Martínez-Solano, 2016).

Posteriormente, (Montanes, et al., 2018) han seguido investigando sobre la aplicabilidad de esta metodología, empleándola en el Grado en Ingeniería en Diseño Industrial y Desarrollo de Productos, impartida en la UPV. Estos profesores han demostrado que la implantación de un método de DI en su asignatura ha incrementado el rendimiento académico, junto con una reducción del porcentaje de "no aptos" a apenas un $2 \%$. Por otro lado, el nivel de satisfacción general de los alumnos se ha incrementado en más de 1 punto respecto a cuando el método docente que se empleaba eran las clases magistrales.

Además, estos trabajos previos, por otro lado, han evidenciado que aún es pronto para establecer conclusiones sólidas y que sigue siendo necesario investigar sobre las barreras existentes y las debilidades del proceso que impiden la implantación y difusión de esta metodología. Asimismo, es necesario identificar los factores de éxito que pueden fomentar la implantación de esta metodología innovadora y potencialmente beneficiosa para el proceso de aprendizaje.

En este contexto, la aportación de esta investigación reside en fomentar la difusión de la metodología de aprendizaje de DI que, literalmente, invierte el concepto tradicional de clase y pone en el centro del 
proceso de aprendizaje el alumno. El rol del profesor es el de fomentar el interés del alumnado y despertar su curiosidad a través del posible uso de herramientas multimedia antes de la clase. De esta forma se incrementa el compromiso del alumno para crear su propio conocimiento. Además, complementaria con esta primera fase de trabajo, principalmente individual, el proceso prevé un trabajo grupal, de cooperación y colaboración entre los alumnos que se podrá realizar en aula o bien a través de herramienta TIC como chat, foros, etc. (Universitat Politécnica de Valéncia, 2015-2020). Además de esto, la presente investigación pretende explorar e investigar la predisposición del alumnado hacia esta metodología, la cual se ha estado aplicando durante algunos años en varias asignaturas de la UPV. Sobre todo, se pretende averiguar el grado de concienciación del alumnado sobre las ventajas que su implantación puede conllevar, entre ellas (Universitat Politécnica de Valéncia, 2015-2020):

- Aprendizaje con máximo aprovechamiento del tiempo en aula

- Personalización del ritmo de estudio de cada alumno y del aprendizaje

- Reducción del absentismo en aula, ya que el alumnado deberá defender su trabajo

- Aumento del compromiso por parte del alumnado debido a la posibilidad de disponer del material didáctico en línea

- Aumentar el trabajo colaborativo y de equipo

- Aprender más y mejor

El estudio se ha basado en una muestra de alumnos de $3^{\circ}$ curso de grado y $1^{\circ}$ curso de máster, correspondientes a las titulaciones de Grado en Ingeniería Eléctrica, Grado en Ingeniería Mecánica y Máster Universitario en Ingeniería Industrial de la UPV.

El artículo se presenta estructurado de la siguiente forma: tras una breve introducción sobre la metodología de docencia inversa, en el capítulo 2 se presentan los objetivos que se pretende alcanzar. En el capítulo 3 se describe el desarrollo de la innovación, incluyendo una contextualización del ámbito académico en la cual se ha llevado a cabo. Después de realizar una exposición de la metodología aplicada, el capítulo 4 presenta los resultados obtenidos. Finalmente, el trabajo se cierra con las conclusiones que se exponen en el capítulo 5 .

\section{Objetivos}

El objetivo principal del presente trabajo es investigar el nivel de aceptación de la metodología de docencia inversa y el grado de concienciación acerca de los beneficios de su implementación entre los alumnos de la UPV. Este objetivo principal se desarrolla a través de los siguientes objetivos específicos:

- Evaluar el nivel de predisposición del alumnado hacia una metodología de docencia innovadora.

- Valorar la importancia del uso de las herramientas TIC para fomentar el aprendizaje.

- Ponderar el conocimiento adquirido a lo largo del curso de estudio sobre la metodología de docencia inversa.

- Ponderar la importancia que las diferentes fases de la metodología de docencia inversa tienen para el alumno.

\section{Desarrollo de la innovación}

\subsection{Contexto académico}

El proyecto de DI se inició en la UPV en 2014 y fue impulsado principalmente por el Vicerrectorado de Estudios, Calidad y Acreditación junto con el ASIC y el ICE. Desde entonces, el proyecto en general ha 
ido creciendo, difundiéndose entre los profesores y las distintas asignaturas con éxito. La Tabla 1 muestra el estado de participación en el proyecto de Docencia Inversa por parte del profesorado de la UPV y el número de asignaturas que han utilizado este tipo de metodología durante los últimos dos años (2018-2019).

Tabla 1. Número de profesores y asignaturas donde se imparte docencia inversa en la UPV

\begin{tabular}{|c|c|c|c|c|}
\hline & \multicolumn{2}{|c|}{2018} & \multicolumn{2}{c|}{2019} \\
\hline EPSA & Profesores & Asignaturas & Profesores & Asignaturas \\
\hline EPSG & 38 & 49 & 27 & 39 \\
\hline ETSA & 38 & 52 & 25 & 35 \\
\hline ETSEAMN & 42 & 21 & 9 & 15 \\
\hline ETSICCP & 22 & 45 & 26 & 26 \\
\hline ETSID & 55 & 30 & 19 & 22 \\
\hline ETSIE & 9 & 10 & 37 & 44 \\
\hline ETSIGCT & 27 & 27 & 4 & 7 \\
\hline ETSII & 37 & 37 & 49 & 17 \\
\hline ETSINF & 42 & 37 & 23 & 42 \\
\hline ETSIT & 11 & 10 & 12 & 22 \\
\hline FADE & 28 & 22 & 10 & 8 \\
\hline FBA & 24 & 24 & 19 & 12 \\
\hline MASTER & 11 & 8 & 6 & 18 \\
\hline & 414 & 418 & 288 & 13 \\
\hline
\end{tabular}

Fuente: VI Jornada de Docencia Inversa en el Campus de Vera 13 de marzo 2019, https://docenciainversa.blogs.upv.es/] y elaboración propia.

Como se puede observar, en este último año se ha detectado una caída en la participación por parte del profesorado, de alrededor el $20 \%$. Este fenómeno no se asocia a una falta de interés en la metodología por parte del profesorado, sino que se debe a un cambio en el sistema de reconocimiento de los créditos académicos que ha afectado negativamente la participación. A pesar de esto, de acuerdo al Flipped Learning Ranking (https://flglobal.org/), el número de profesores y asignaturas que participan en el proyecto de DI sigue siendo de la más altas entre las universidades de España.

Como muestran los dados de la encuesta del año 2019, en la que han participado 151 profesores por 146 asignaturas, la metodología de la DI en la UPV se aplica, principalmente, en cursos de máster (25\%) y en el $4^{\circ}$ curso de grado $(20 \%)$, en asignaturas con un tamaño de grupo pequeño-mediano de entre 10 y 30 alumnos, tal y como se muestra en la Figura 1 . Esta tendencia está justificada por el hecho que la implantación de la metodología de DI implica un esfuerzo muy significativo por parte del profesorado. Alrededor del $80 \%$ del profesorado afirma haber tenido que generar nuevos materiales docentes (vídeos en Poliformat, polimedias, screencast, vídeos didácticos) o haber tenido que hacer una nueva programación de la asignatura para adaptarla a esta nueva metodología. A pesar de esto, los motivos principales que han impulsado a estos profesores a implantar una metodología de DI en su asignatura han sido, en orden de importancia y de acuerdo a los resultados de la encuesta del 2019-2020, los siguientes:

- $\quad$ El interés en mejorar la docencia e innovarla (85\%)

- Fomentar la participación activa de los alumnos en clase (23\%)

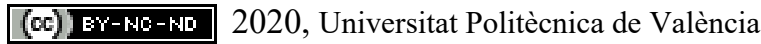

Congreso In-Red (2020) 
- Otros motivos son la poca asistencia de los alumnos en clase, el alto índice de suspensos en la asignatura o el alto índice de no presentados en la asignatura.

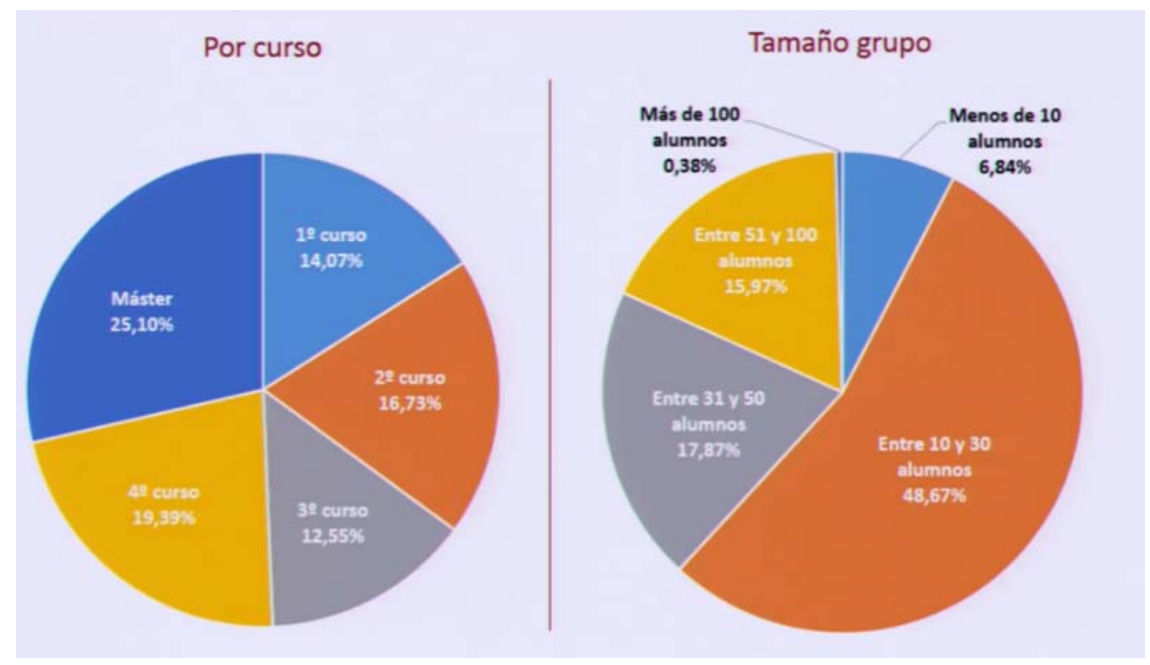

Figura 1. Participación en el programa de DI de la UPV por curso y tamaño de grupo. Fuente: VI Jornada de Docencia Inversa en el Campus de Vera. 13 de marzo 2019 https://docenciainversa.blogs.upv.es/

Finalmente, y siempre desde el punto de vista del profesorado, tras la aplicación de la metodología de docencia inversa se ha detectado que:

- El alumno tiene más autonomía y control sobre su proprio aprendizaje.

- El alumno aprende más y mejor.

Es en este marco, este trabajo se centra en obtener respuesta a preguntas similares, pero desde el punto de vista de los estudiantes de cara a identificar potenciales barreras que obstaculizan la implantación de esta metodología, así como investigar sobre los factores que la impulsan.

\subsection{Metodología}

La metodología utilizada para alcanzar los objetivos de la presente investigación está basada en el diseño y el pase de una encuesta realizada al alumnado antes y después de visualizar un vídeo explicativo sobre la metodología de docencia inversa.

\subsubsection{Descripción del contenido del vídeo explicativo}

El vídeo explicativo que el alumnado ha visualizado ha sido preparado por la UPV ${ }^{1}$. Tiene una duración muy corta de (poco más de 1 minuto) y describe la metodología de docencia inversa desde el punto de vista del alumnado. En el vídeo se detallan las diferentes fases de una clase tipo de docencia inversa, explicitando los diferentes objetivos de aprendizaje.

En el diagrama que se muestra en la Figura 2 (antes de la clase), la Figura 3 (durante la clase) y la Figura 4 (después de la clase), se resume de forma esquemática la planificación de una clase tipo de docencia inversa, tal y como se ha considerado en este trabajo y se muestra en el vídeo.

\footnotetext{
${ }^{1}$ El vídeo está disponible en esta dirección web: https://media.upv.es/player/embed.html?id=baa8de70-2692-11e8-b43a-51b816915a74).4
} 


\subsubsection{Metodología de pase de encuesta}

La metodología utilizada se resume en la Figura 5, donde se muestran las dos fases en las que se ha dividido el proceso de encuesta: uno antes de la visualización del vídeo y otro posterior tras su visualización.

\section{FASE 1: ANTES DE LA CLASE}

1 Leer el tema que se va a tratar cómodamente en casa

Visionar el vídeo explicativo proporcionado por el profesor todas las veces que se considere necesario

\section{OBJETIVOS DE APRENDIZAJE:}

- Comprender el tema que se va a tratar en clase

- Identificar los pasos a seguir para resolver el problema

- Investigar sobre el tema y los diferentes caminos para resolverlo

- Conocer los diferentes caminos para resolver el problema

Figura 2. Procedimiento de Docencia Inversa antes de la clase.

\section{DURANTE LA CLASE}

FASE 2: Trabajo en equipo del alumnado y puesta en común de los conocimientos adquiridos en la fase 1.

FASE 3: Resolución de dudas por parte del profesor (feedback inmediato)

\section{OBJETIVOS DE APRENDIZAJE:}

\section{- Analizar el problema}

Aplicar los conocimientos adquiridos en casa

Crear una estrategia para resolver el problema

Diseñar la solución del problema

Figura 3. Procedimiento de Docencia Inversa durante de la clase. 


\section{TRAS LA CLASE}

\section{FASE 4: Evaluación}

FASE 5: Resumen y actividades de consolidación

\section{OBJETIVOS DE APRENDIZAJE:}

\section{- Consolidar conceptos aprendidos}

Figura 4. Procedimiento de Docencia Inversa después de la clase.

a) Antes de la visualización del vídeo

La primera fase se desarrolla antes de la visualización del vídeo por parte de alumnado. En esta fase se evalúa, primero, el conocimiento previo de los estudiantes acerca de la docencia inversa. Después, se evalúa la predisposición del alumnado a experimentar nuevas metodologías docentes. Finalmente, se valora la importancia del uso de herramientas TIC por parte del alumnado.

b) Después de la visualización del vídeo

La segunda parte de la encuesta se ha desarrollado tras haber mostrado a los alumnos el vídeo informativo preparado por la UPV. En esta segunda fase se presenta a los alumnos la metodología de docencia inversa y cómo se desarrolla a través de sus distintas fases:

- FASE 1: El alumno realizará un trabajo previo en casa (antes de la clase) orientado por el profesorado

- FASE 2: Durante la clase, el alumno realizará el trabajo de forma colaborativa con sus compañeros, y se expondrán las dudas al profesor.

- FASE 3: El profesor resolverá en aula las dudas de los alumnos (feedback inmediato).

- FASE 4 y 5: Las fases de evaluación y de consolidación de los conceptos aprendidos no forman parte del alcance de este artículo.

A través de esta encuesta, se identifica la importancia que el alumno da a cada una de las fases de una clase de docencia inversa y se investiga su grado de aceptación de esta técnica docente. Finalmente se pregunta al alumno si le gustaría que en más clases se adoptara la docencia inversa. 
Antes de visionar el vídeo

Conocimientos previos sobre de la docencia inversa

\section{Predisposición del alumnado a experimentar metodologías de} docencia innovadora

\section{Herramientas de aprendizaje TIC}

\section{Después de visionar el vídeo}

Valoración de la importancia de las diferentes fases de la metodología de docencia inversa

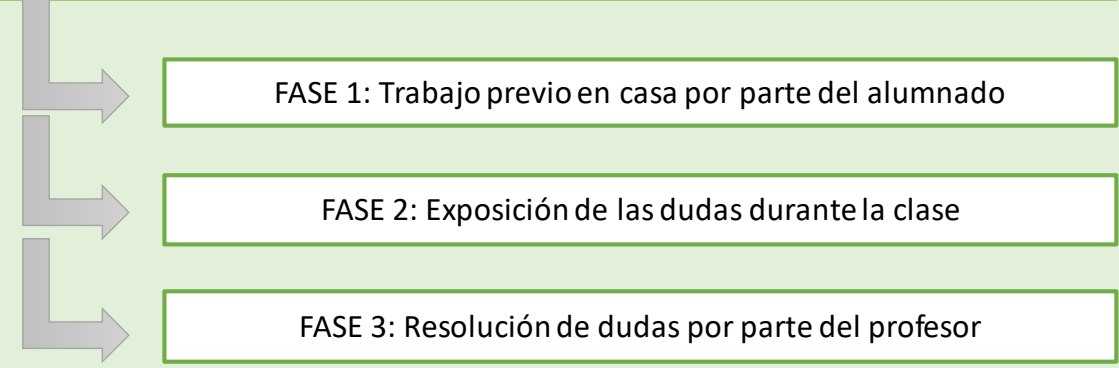

Figura 5. Metodología utilizada durante la encuesta a los alumnos

\section{Resultados}

En este capítulo se presenta el caso de aplicación de la metodología y se analizan los resultados obtenidos en la encuesta. La encuesta, en su formato integral, se puede consultar en el Anexo 1 de este documento.

\subsection{Caso de aplicación de la metodología: selección de la muestra}

Para la realización de la encuesta, se ha elegido una muestra de alumnos de $3^{\circ}$ curso de grado y $1^{\circ}$ curso de máster, correspondientes a las titulaciones de Grado en Ingeniería Eléctrica, Grado en Ingeniería Mecánica y Máster Universitario en Ingeniería Industrial. 
La elección de este segmento se basa en la necesidad de que los alumnos hayan podido cursar asignaturas impartidas a través de la metodología de docencia inversa. Estos alumnos se concentran en este segmento, tal y como se menciona en el apartado 3.1 'contexto académico'. De esta forma, se tiene la mayor probabilidad que los alumnos conozcan o hayan cursado asignaturas de docencia inversa y poder, de esta manera, detectar con más agilidad las razones que pueden ayudar fomentar la difusión de esta metodología. No se han tenido en cuenta edad, género, calificaciones o intereses de los alumnos.

Una vez seleccionado el segmentado, se ha elegido el subgrupo representativo de la muestra, que corresponde a una población equivalente al $20 \%$ del total de los alumnos matriculados en el $3^{\circ}$ curso de grado y $1^{\circ}$ de postgrado de las titulaciones consideradas. De acuerdo con estudios previos, una muestra representativa debe ser equivalente a un 20-30 \% del total de la población para así poder generalizar los resultados (García-García, et al., 2013), (Normas APA, 2016). La población de estudiantes de grado de $3^{\circ}$ curso de ingeniería mecánica y eléctrica y primer año de postgrado de ingeniería industrial es para el año 2019-2020 de alrededor de 335 alumnos matriculados, lo que corresponde a un porcentaje que permite generalizar los resultados. La Figura 6 esquematiza el tamaño de la muestra seleccionada, correspondiente a 67 alumnos.

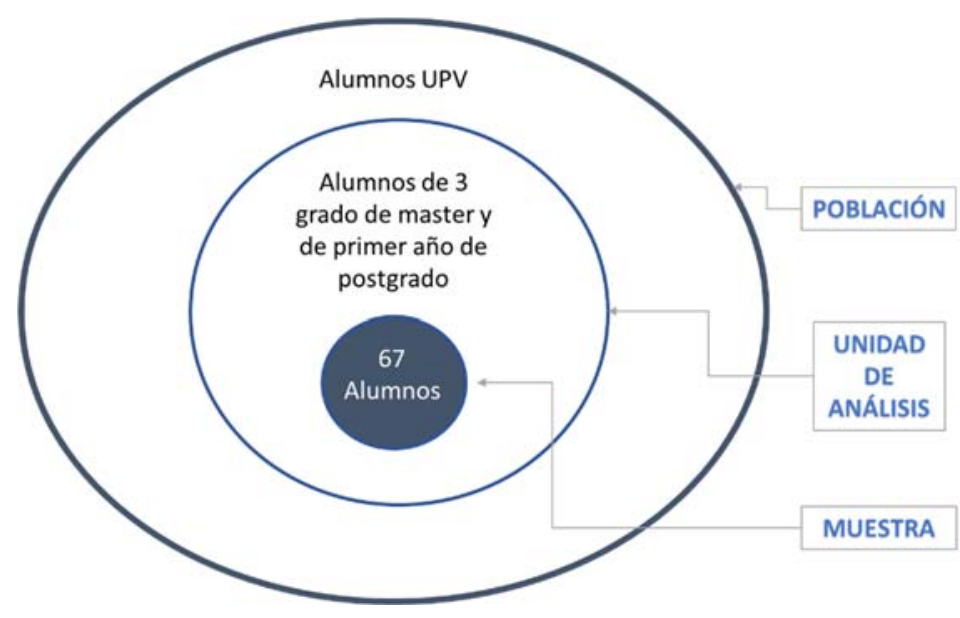

Figura 6. Esquema de selección de la muestra

\subsection{Resultados de la encuesta}

El cuestionario incluido en el Anexo I ha sido utilizado para encuestar a los alumnos de la muestra identificada en el aparatado 4.1., que corresponde a 67 alumnos, distribuidos como se muestra en la Figura 7 entre $3^{\circ}$ curso de grado y $1^{\circ}$ curso de postgrado. Las encuestas de los alumnos de intercambio no han sido tenidas en cuenta, ya que estos alumnos no han tenido la oportunidad de participar en ninguna experiencia de DI en la UPV en los cursos anteriores. 


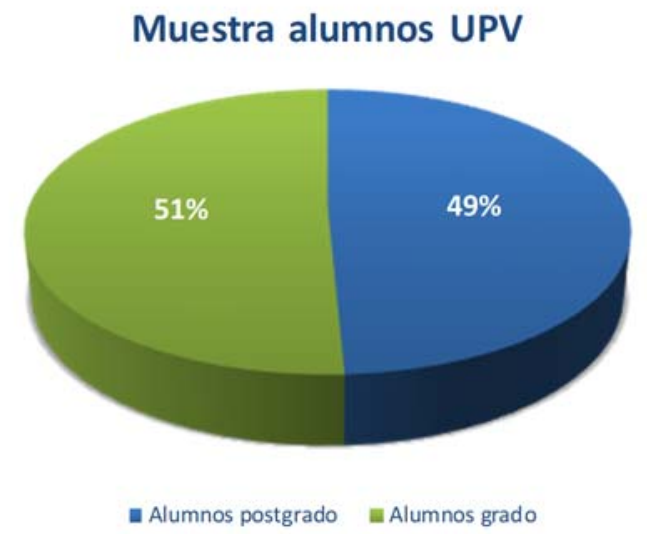

Figura 7. Distribución de los alumnos de la muestra

Las principales conclusiones de la encuesta han sido agrupadas de acuerdo a las dos fases de desarrollo de la metodología, tal y como se presentan a continuación.

a) Antes de la visualización del vídeo

Predisposición del alumnado en experimentar una metodología innovadora de aprendizaje

- Solo el $8 \%$ de los alumnos afirman que leen la teoría antes de la clase mientras que el $51 \%$ no la leen para nada y solo el $41 \%$ de los alumnos afirma leerla un poco antes de la clase.

\section{Causas principales de la falta de interés}

- A pesar de leer la documentación proporcionada por el profesor, el $23 \%$ de los alumnos afirma que no le encuentran ninguna utilidad debido a que las diapositivas son muy esquemática y por sí solas no dicen nada sin el soporte explicativo del profesor. Otros comentan que desconocer cuál es el tema que se va a impartir en la clase debido a falta de planificación por parte del profesor. Finalmente, el 73 \% lamenta no poder leer la teoría antes de la clase por falta de tiempo o de ganas.

\section{Método de clases magistrales}

- El $67 \%$ de los alumnos considera que las clases magistrales representan un método obsoleto, mientras que un $32 \%$ sigue considerando actual e indispensable para su formación.

\section{Clases de prácticas}

- Casi el $100 \%$ de los encuestados manifiesta la necesidad que se programen más clases prácticas y de resolución de problemas prácticos, más que clases teóricas.

\section{Herramientas de aprendizaje}

- Casi el $100 \%$ de los encuestados valora positivamente el uso de material didáctico de tipo multimedia como vídeos, animaciones o actividades interactivas para fomentar el aprendizaje. 
Además, consideran indispensable el uso de aplicaciones informáticas y entre las aplicaciones TIC actualmente en uso y presentes en el mercado el $40 \%$ de los alumnos aconsejan introducir las siguientes en orden de prioridad:
* YouTube
* Instagram
* Twitter
* Polimedia, vídeos
* Kahoot!

\section{Conocimiento previo de la metodología de docencia inversa}

- Con relación al conocimiento previo de la metodología de docencia inversa, destaca que el $73 \%$ ya conoce la docencia inversa y que el $55 \%$ de ellos han cursado entre 1 y 2 dos asignaturas con docencia inversa.

\section{Motivación del profesorado}

- Finalmente, los alumnos confirman que, entre los profesores, el $50 \%$ han trasmitido al alumnado pasión y motivación hacia el proyecto de docencia inversa.

b) Después de visionar el vídeo

Con referencia al modelo de clase de docencia inversa presentado en la sección 3.2, los alumnos han evaluado las diferentes fases:

FASE 1: El alumno realizará un trabajo previo en casa, orientado por el profesorado

FASE 2: Trabajo de equipos y puesta en común de los conocimientos adquiridos en la Fase 1. Además, en esta fase el alumno expondrá sus dudas al profesorado.

FAES 3: El profesor resolverá en aula las dudas de los alumnos (feedback inmediato)

\section{Evaluación del modelo de clase de docencia inversa}

- Casi al $90 \%$ de los alumnos han considerados las tres fases como fundamentales para el proceso de aprendizaje y las valoran positivamente, dando especial importancia a la tercera fase relacionada con el feedback inmediato.

\section{Feedback inmediado}

- Desafortunadamente, el $73 \%$ de los alumnos lamenta no haber recibido un feedback inmediato por parte del profesorado.

Nivel de aceptación de la docencia inversa tras la visualización del vídeo

- $\quad$ El $64 \%$ está de acuerdo con que la docencia inversa permite aprender mejor

- El $94 \%$ considera que la docencia inversa aumenta el trabajo en casa del alumnado

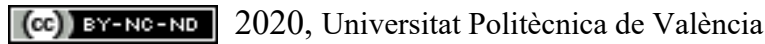

Congreso In-Red (2020) 
- El $83 \%$ ve en la docencia inversa como una metodología que deja solo al alumno en el proceso de aprendizaje

- $\quad$ El $74 \%$ considera que se remplaza los profesores con vídeos.

- El $76 \%$ considera que la docencia inversa representa un nuevo enfoque de aprendizaje que permite evitar consumir el tiempo con la teoría.

\section{Fomento de la difusión de la docencia inversa entre los alumnos}

- Tras la visualización del vídeo, solo el $36 \%$ del alumnado manifiesta el deseo de participar en un curso de docencia inversa.

\section{Conclusiones}

La investigación que se presenta en este artículo evidencia una reticencia todavía muy alta por parte del alumnado a aceptar la docencia inversa. A pesar de la que los efectos positivos en términos de mejoras del aprendizaje que la docencia inversa puede conllevar están asumidos, los alumnos consideran muy alta la carga de trabajo adicional en casa que esta metodología implica antes de la clase. Existen aún muchos prejuicios en el alumnado hacia la docencia inversa, sobre todo en lo referente a que el estudiante tenga que trabajar solo en casa, así como en la consideración de que el profesor es simplemente reemplazo por un vídeo.

Del análisis realizado se deduce que los alumnos no están acostumbrados a desarrollar actividades preparatorias a la clase, ya sea por falta de organización o de motivación. Por lo tanto, los profesores de hoy en día tienen el reto de estimular y motivar el interés del alumnado, ayudándoles a mejorar su capacidad de gestionar el tiempo. Los alumnos demuestran tener predisposición para implantación de nuevas metodologías de aprendizaje, sobre todo cuando están relacionadas con la introducción de material multimedia, y piensan que las clases prácticas y con soluciones TIC tienen que ir ganando protagonismo frente a las clases magistrales tradicionales.

Finalmente, el profesorado debería potenciar su trabajo intentando proporcionar a los alumnos feedback inmediatos o, al menos, en un tiempo reducido, de forma que puedan alcanzar los objetivos de aprendizaje de manera más adecuada y puedan asimismo progresar en su desarrollo académico.

\section{Agradecimientos}

Este trabajo ha sido respaldado en parte por la administración pública de la Generalitat Valencia bajo la beca ACIF/2018/106.

\section{Referencias}

Argente, E., Espinosa, A. \& García-Fornés, A., 2015. Experiencia de la aplicación de la metodología Flipped-Teaching en la asignatura Concurrencia y Sistemas Distribuidos. Congreso In-Red, Universitat Politècnica de València.

Aulaplaneta, 2015. Clintondale High School: la primera escuela en poner todas sus clases del revés con la flipped classroom. [En línea] Available at: https://www.aulaplaneta.com [Último acceso: marzo 2020]. 
Bonet, P. y otros, 2015. Proyecto Competencias Transversales UPV, Valencia: Universitat Politècnica de València.

Christensen, C. R., Garvin, D. A. \& Sweet, A., 1992. Education for judgement. The artistry of discussion leadership. ISBN 9780875843650. Boston: Harvard Business Review Press.

García-García, J. A., Reding-Bernal, A. \& López-Alvarenga, J. C., 2013. Cálculo del tamaño de la muestra en investigación en educación médica. Metodología de investigación en educación médica, 2(8), pp. 217-224.

Llácer-Iglesias, R. \& Martínez-Solano, F. J., 2016. Experiencia en la aplicación de la docencia inversa para el aprendizaje de la asignatura de Máquinas Hidráulicas (Grado de Ingeniería en Tecnologías Industriales). Valencia, 7-8 julio, Universitat Politécnica de Valéncia.

Montanes, N. y otros, 2018. ¿Por qué es importante aplicar docencia inversa? ¿Cómo hacerlo? Valencia (España), 19-20 de julio, Universitat Politècnica de València, pp. 551-562.

Normas APA, 2016. ¿Qué es una muestra y cómo se selecciona? [En línea] Available at: http://normasapa.net/que-es-una-muestra-y-como-se-selecciona/ [Último acceso: marzo 2020].

Roach, T., 2014. Student perceptions toward flipped learning: new methods to increase interaction and active learning in economics. International Review of Economics Education, Volumen 17, pp. 74-84.

The Flipped Classroom, 2015. ¿Qué es Flipped-Classroom?. [En línea] Available at: https://www.theflippedclassroom.es/what-is-innovacion-educativa/ [Último acceso: March 2020].

Universitat Politécnica de Valéncia, 2015-2020. Proyecto de Docencia Inversa en la UPV. [En línea] Available at: https://docenciainversa.blogs.upv.es/ [Último acceso: marzo 2020]. 


\section{Anexo. Cuestionario}

\section{ENCUESTA SOBRE DOCENCIA INVERSA EN LA UPV}

\begin{tabular}{|c|l|}
\hline a) Indica tu año de curso & Respuesta abierta \\
\hline b) ¿Eres un alumno de intercambio? & Respuesta dicotómica \\
\hline
\end{tabular}

\section{* Antes de la visualización del vídeo}

\section{Primera Parte: Predisposición del alumnado}

\begin{tabular}{l|l}
\hline 1) ¿Lees la teoría antes de la clase? & Escala Likert
\end{tabular}

2) Cuando la has leído, ¿te ha ayudado?

3) Si no la lees, ¿cuál es la razón?

Escala Likert

Respuesta abierta

¿Cuánto estás de acuerdo con las siguientes afirmaciones?:

4) "Las clases magistrales han quedado obsoletas" $\quad$ Escala Likert

5) "El uso de material multimedia y audiovisual (ej. vídeos) favorece el $\quad$ Escala Likert aprendizaje"

6) "Las horas de clase deberían ser empleadas para hacer más prácticas y Escala Likert ejercicios en lugar de explicar teoría"

Segunda Parte: Conocimiento de la Docencia Inversa

7) ¿Conoces qué es la docencia inversa?

8) Durante tus estudios universitarios: ¿has tenido profesores que han aplicado la docencia inversa?

9) Indica en cuántas asignaturas se ha utilizado la docencia inversa

10) El profesor que ha impartido las clases de docencia inversa: ¿estaba motivado para ello?

\begin{tabular}{|l|l|}
\hline \multicolumn{2}{|l|}{ Tercera Parte: Herramienta de aprendizaje } \\
\hline Valora cuánto consideras que sea importante para fomentar el aprendizaje que: \\
\hline $\begin{array}{l}\text { 11) } \\
\text { El material didáctico de una asignatura sea multimedia e interactivo } \\
\text { (vídeos, animaciones y actividades) }\end{array}$ & Escala Likert \\
\hline 12) Se utilicen aplicaciones informáticas como Kahoot, Socratis, Lessons & Escala Likert \\
\hline $\begin{array}{l}\text { 13) Sugiere qué aplicaciones consideras se debería introducir el } \\
\text { profesorado para fomentar el aprendizaje (ej. Instagram; Twitter; } \\
\text { Facebook, etc.) }\end{array}$ & Respuesta abierta \\
\hline
\end{tabular}




\section{* Tras la visualización del vídeo}

Vídeo para el alumno

https://media.upv.es/player/embed.html?id=baa8de70-2692-11e8-b43a-51b816915a74

\begin{tabular}{|l|l|}
\hline \multicolumn{2}{|l|}{ Valora la importancia de cada fase de este método: } \\
\hline 14) $\begin{array}{l}\text { FASE 1: El alumno realizará un trabajo previo en casa (antes de } \\
\text { la clase) orientado por el profesorado }\end{array}$ & Escala Likert \\
\hline $\begin{array}{l}\text { 15) } \\
\text { FASE 2: Tras la realización del trabajo, el alumno expondrá sus } \\
\text { dudas al profesorado durante la clase }\end{array}$ & Escala Likert \\
\hline $\begin{array}{l}\text { 16) FASE 3: El profesor resolverá en aula las dudas de los alumnos } \\
\text { (feedback inmediato) }\end{array}$ & Escala Likert \\
\hline
\end{tabular}

\section{Indica cuánto estás de acuerdo con las siguientes afirmaciones:}

\begin{tabular}{|c|c|}
\hline $\begin{array}{l}\text { 17) "La docencia inversa permite aprender más y mejor que la } \\
\text { docencia tradicional" }\end{array}$ & Escala Likert \\
\hline 18) "La docencia inversa aumenta el trabajo en casa del alumnado" & Escala Likert \\
\hline $\begin{array}{l}\text { 19) "La docencia inversa deja solo al alumnado en el proceso de } \\
\text { aprendizaje en casa" }\end{array}$ & Escala Likert \\
\hline $\begin{array}{l}\text { 20) "La docencia inversa es sinónimo de curso en línea con } \\
\text { reemplazo del profesor por vídeos" }\end{array}$ & Escala Likert \\
\hline $\begin{array}{l}\text { 21) "La docencia inversa evita tener que consumir el tiempo de clase } \\
\text { en explicaciones de teoría" }\end{array}$ & Escala Likert \\
\hline
\end{tabular}

22) A lo largo del curso, valora el porcentaje de profesores que han dado feedback inmediato.

23) ¿Te gustaría que la docencia inversa se impartiera en tus asignaturas? 